\title{
Health threat from cosmic radiation during manned missions to Mars
}

\author{
Alexandra D. Bloshenko, ${ }^{a, *}$ Jasmin M. Robinson, ${ }^{a}$ Rafael A. Colon ${ }^{b}$ and \\ Luis A. Anchordoqui ${ }^{a}$ \\ ${ }^{a}$ Department of Physics \& Astronomy, Lehman College, CUNY, NY 10468, USA \\ ${ }^{b}$ Weill Cornell Medicine, Cornell University, NY 10065, USA \\ E-mail: sasha.bloshenko@gmail.com
}

\begin{abstract}
Cosmic radiation is a critical factor for astronauts' safety in the context of evaluating the prospect of future space exploration. The Radiation Assessment Detector (RAD) on board the Curiosity Rover launched by the Mars Scientific Laboratory mission collected valuable data to model the energetic particle radiation environment inside a spacecraft during travel from Earth to Mars, and is currently doing the same on the surface of Mars itself. The Martian Radiation Experiment (MARIE) on board the Mars Odyssey satellite provides estimates of the absorbed radiation dose in the martian orbit, which are predicted to be similar to the radiation dose on Mars' surface. In combination, these data provide a reliable assessment of the radiation hazards for a manned mission to Mars. Using data from RAD and MARIE we reexamine the risks for a crew on a manned flight to Mars and discuss recent developments in space exploration.
\end{abstract}

$37^{\text {th }}$ International Cosmic Ray Conference (ICRC 2021)

July 12th - 23rd, 2021

Online - Berlin, Germany

\footnotetext{
${ }^{*}$ Presenter
} 


\section{Introduction}

Space - the final frontier. It has almost become widely accepted that it is our destiny to explore strange new worlds, to seek out new life and new civilizations, to boldly go where no one has gone before. However, humanity will have to find new ways to adapt to the cold harsh environments of outer space if it is to ever become a space-faring civilization.

One of the greatest threats to long term human survival during spaceflight is the exposure to high energy radiation. There are two primary types of radiation that humans are exposed to in space: solar energetic particle (SEP) events and Galactic cosmic rays (GCRs) [1]. Of the two, GCRs are a chronic source of high energy radiation that may damage the DNA of humans exposed to them for long periods of time, whereas the levels of damage caused by SEPs may vary drastically with the strength, duration, and location of the solar event [2]. The GCR flux fills the interplanetary space and is made up of about $85 \%$ hydrogen nuclei, $14 \%$ helium nuclei, and around 1\% high-energy and highly charged $(Z>2)$ nuclei referred to as HZE particles [3]. Though there are fewer HZE particles than there are hydrogen and helium nuclei, they possess significantly higher ionizing power, greater penetration power, and a greater potential for the radiation-induced damage to DNA. Therefore, determining the absorbed radiation dosage of GCRs and SEPs that an astronaut would receive during a manned mission to Mars is necessary prior to any such mission. In this paper we reexamine the radiation sources and risks for a crew on a manned flight to Mars.

One important factor of our study is the duration of the trip to Mars. There are short duration (roughly 600 days with 30-day Mars stay time) or long stay time (about 550 days with 180-day transfer to Mars and 180-day inbound transfer) missions [4]. The usual benchmark here is the so-called Hohmann transfer, which is an elliptical orbit used to transfer between the two circular planet orbits using the lowest possible amount of propellant. The launch from Earth for a successful Hohmann transfer must occur when Earth is at perihelion and the landing takes place when Mars is at aphelion. When launched within the proper window, a spacecraft will reach Mars' orbit just as the planet moves to the same point. Astronauts would travel from Earth to Mars, wait until the next synchronized alignment between the planets (about 460 days), and start the return trip to Earth. Kepler's 3rd law,

$$
(\text { orbital period } / \mathrm{yr})^{2}=(\text { Hohmann orbit semi-major axis } / \mathrm{AU})^{3},
$$

provides a way to estimate the time-scale for the trip to Mars. Since the mean Earth-Sun distance is $\bar{D}_{\odot-\oplus}=1 \mathrm{AU}$ and the mean Mars-Sun distance is $\bar{D}_{\odot-\odot} \simeq 1.542 \mathrm{AU}$, the Hohmann orbit semi-major axis $=\left(\bar{D}_{\odot-\oplus}+\bar{D}_{\odot-\odot}\right) / 2 \simeq 1.262 \mathrm{AU}$. Therefore, the interplanetary travel will take 259 days each way, yielding a total mission time of 978 days. We will use this time-scale to estimate the radiation hazards for a manned mission to Mars.

Before proceeding, we pause to specify and establish the relationship between the four different units used to measure the amount of radiation absorbed by an object or person, known as the "absorbed dose." Such an absorbed dosed reflects the amount of energy that radioactive sources deposit in materials through which they pass. The radiation absorbed dose (rad) is the amount of energy (from any type of ionizing radiation) deposited in any medium (e.g., water, tissue, air). An absorbed dose of $1 \mathrm{rad}$ means that 1 gram of material absorbed 100 erg of energy as a result of exposure to radiation. The related international system unit is the gray (Gy), where $1 \mathrm{~Gy}$ is 
equivalent to $100 \mathrm{rad}$. Another relevant unit is the Sievert ( $\mathrm{Sv}$ ), which is an ionizing radiation dose that measures the amount of energy absorbed by a human body per unit mass $(\mathrm{J} / \mathrm{kg})$. This biological unit relates to the previous units according to: $1 \mathrm{~Sv}=1 \mathrm{~Gy}=100 \mathrm{rad}$. Finally, the fourth important unit to define is the Roentgen equivalent man (rem), which represents the dosage in rads that will cause the same amount of biological injury as one rad of X-rays or $\gamma$-rays: $1 \mathrm{~Sv}=100 \mathrm{rem}$.

\section{Radiation hazards on space missions}

High-energy $(\gtrsim \mathrm{GeV})$ protons and nuclei penetrating the spacecraft will be subjected to different atomic and nuclear processes. These relativistic particles will suffer inelastic collisions that produce, on average, a number of fast secondary hadrons. Some of these hadrons (protons, neutrons, charged pions) will have further nuclear collisions, resulting in a hadronic cascade. Neutral pions, however, will decay almost instantly and their decay products ( $\gamma$-pairs) can initiate electromagnetic showers, which are sustained by: $(i)$ electron-positron pair production by photons; (ii) Bremsstrahlung losses of electrons and positrons. As the particle energy decreases, other processes become dominant (Compton scattering of photons, photoelectric effect, ionization losses of charged particles). Some of the charged pions and other hadrons will also decay and produce muons. Besides the production of fast hadrons, nuclear collisions also generate lower-energy $(\mathrm{MeV})$ neutrons, protons, light ions (alpha particles) and gamma rays, which are emitted during the de-excitation of target nuclei. The protons and light ions

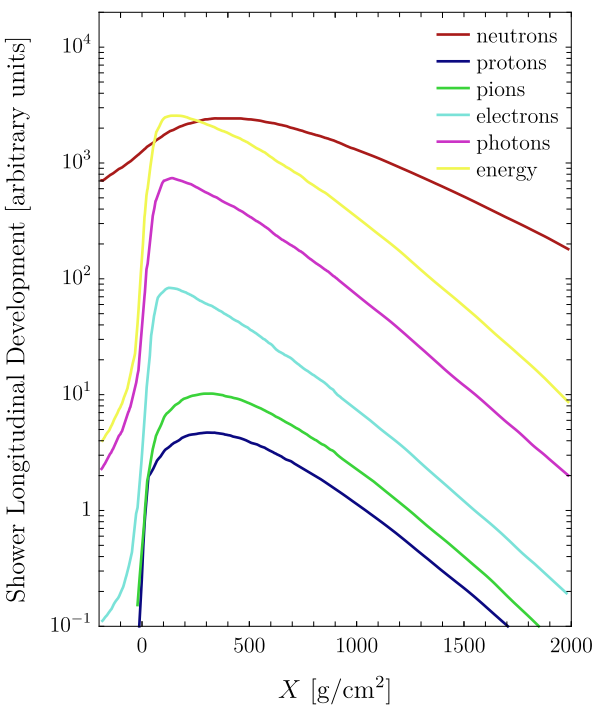

Figure 1: Longitudinal development of a $100 \mathrm{GeV}$ proton shower on lead as a function of depth $X$. will mainly range out because of ionizing losses. The neutrons, however, can be very penetrating and fully capable of depositing a damaging dose deep inside tissue. As an illustration of all these processes, in Fig. 1 we show the longitudinal development of a simulated $100 \mathrm{GeV}$ proton shower on lead, using the program FLUKA.

The overwhelming health risk comes from GCRs since they exist as an isotropic background and are, therefore, consistently dangerous throughout the entire mission rather than at random intervals whenever SEP events occur. The energetic cosmic particles would strike the spacecraft to initiate hadronic cascades. The thicker the material they pass through, the more secondary particles there will be in the cascade. In condensed matter (liquid or solid) the shower of a $1 \mathrm{GeV}$ proton continues to grow until it reaches about $200 \mathrm{~g} / \mathrm{cm}^{2}$, which is the equivalent of about 2 meters of water. After this point the shower begins to attenuate. The attenuation is exponential, with a $1 / e$ attenuation length of about $200 \mathrm{~g} / \mathrm{cm}^{2}$. The attenuation length in the Earth's atmosphere is somewhat smaller, roughly $140 \mathrm{~g} / \mathrm{cm}^{2}$. In a gas, like in the Earth's atmosphere, the dependency of the average depth of the peak particle intensity $X_{\max }$ on energy can be parametrized as $\left\langle X_{\max }\right\rangle=a+b \log _{10}(E / \mathrm{GeV})$, where $E$ is the energy of the primary particle [5]. For simulated $\gamma$-ray showers, $a=98 \mathrm{~g} / \mathrm{cm}^{2}$ 
and $b=83 \mathrm{~g} / \mathrm{cm}^{2}$, whereas for proton primaries $a=111 \mathrm{~g} / \mathrm{cm}^{2}$ and $b=74 \mathrm{~g} / \mathrm{cm}^{2}$ [6]. Whether pions decay or initiate more interactions accounts for the attenuation difference between condensed matter and gas.

In the outer heliosphere, beyond about $100 \mathrm{AU}$, the slowing of solar wind is thought to form a large magnetic barrier that shields out $\gtrsim 90 \%$ of the GCR radiation present in interstellar space at energies below roughly $100 \mathrm{MeV}$ [7]. Because this reduction is so large, even a very small change in the shielding efficiency can have a large impact on the radiation environment in the solar system. However, because these regions have never been directly sampled or observed, there is great uncertainty about the physics of outer heliospheric shielding and its sensitivity to changes in the solar wind output and the local interstellar medium. A small fraction of GCRs penetrate into the heliosphere and propagate toward the Sun and planets. These residual GCRs are modulated by the solar wind's magnetic field in the inner heliosphere [8,9]. The GCR intensity is at its lowest during the peak of solar activity. This is because the enhanced solar activity sweeps away the low energy part of the GCR spectrum. The effect is statistically significant and more than compensates for solar generated cosmic rays during solar maximum.

Focusing now directly on Mars, the red planet lost its magnetosphere 4 billion years ago and thus the solar wind interacts directly with the martian ionosphere, lowering the atmospheric density by stripping away atoms from the outer layer. The atmosphere of Mars consists of about $95 \%$ carbon dioxide, $3 \%$ nitrogen, $1.6 \%$ argon and contains traces of oxygen, water, and methane [10]. The scale height ( $\equiv$ vertical distance over which the density and pressure fall by a factor of $1 / e$ ) of the martian atmosphere is about $10.8 \mathrm{~km}$, which is higher than Earth's $(8.5 \mathrm{~km})$; the surface gravity of Mars is only about 38\% of Earth's, an effect offset by both the lower temperature and 50\% higher average molecular weight of the atmosphere of Mars. The atmospheric pressure on the surface today ranges from a low of $0.030 \mathrm{kPa}$ on Olympus Mons to over $1.155 \mathrm{kPa}$ in Hellas Planitia, with a mean pressure at the surface level of $0.60 \mathrm{kPa}$ (which is only $0.6 \%$ of that of the Earth $101.3 \mathrm{kPa}$ ). The highest atmospheric density on Mars is equal to the density found $35 \mathrm{~km}$ above the Earth's surface. However, the atmosphere of Mars is just thick enough to produce plenty of dangerous secondaries (including neutrons) from GCRs and SEPs. The radiation would be reduced to Earth-like intensities for facilities located at a depth of about $2,000 \mathrm{~g} / \mathrm{cm}^{2}$ beneath the martian surface.

In our analysis we use data from the Radiation Assessment Detector (RAD) on board Curiosity Rover launched by the Mars Scientific Laboratory (MSL) mission [11-13] and the Martian Radiation Environment Experiment (MARIE) on board the Mars Odyssey satellite [14]. The RAD data collection instrument gathered valuable data to model the energetic particle radiation environment inside a spacecraft during travel from Earth to Mars [11, 12], and is currently doing the same on the surface of Mars itself [13]. The MARIE data collection instrument provides estimates of the absorbed radiation dose in the martian orbit, which are predicted to be similar to the absorbed radiation dose on the surface of Mars [14]. Variations of the GCR absorbed radiation dose rate during the transfer between Earth and Mars range from 1.75 to $3.0 \mathrm{mSv} /$ day [11, 12]. The GCR absorbed radiation dose on the surface of Mars measured by the RAD instrument is $0.21 \mathrm{mGy} /$ day [13]. The GCR absorbed radiation dose in the martian atmosphere (collected by the MARIE instrument) is $0.25 \mathrm{mGy} /$ day [14]. In Fig. 2 we show the estimated radiation dose from GCRs on the martian surface, using data from MARIE and the Mars Orbiter Laser Altimeter (MOLA) (which was one of five instruments on the Mars Global Surveyor spacecraft that operated in Mars orbit from September 

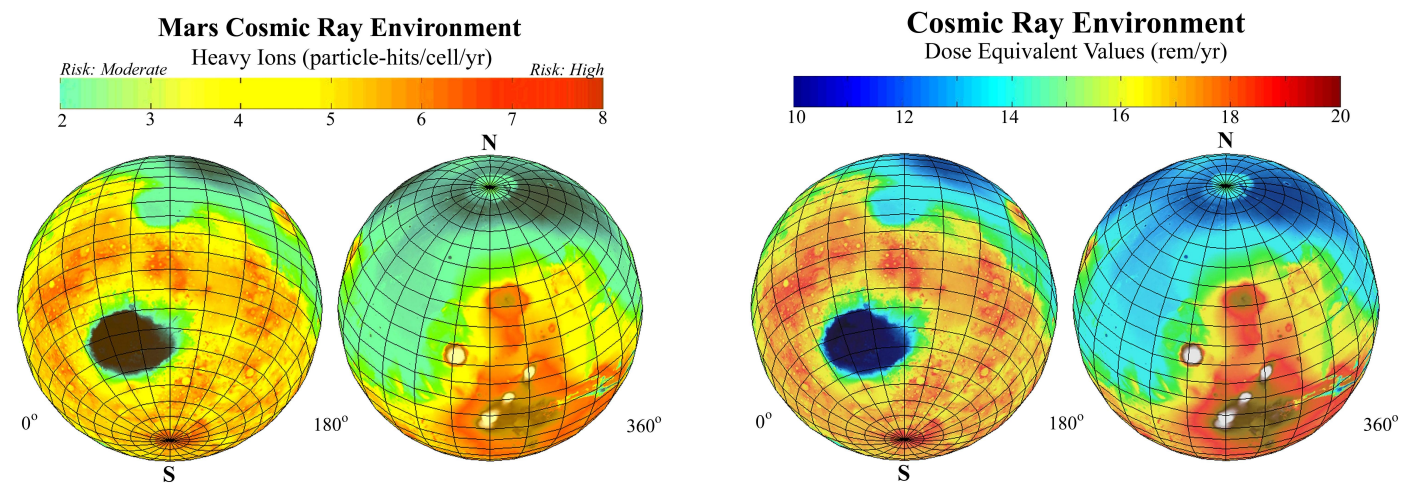

Figure 2: Estimated radiation dose from GCRs on the martian surface, from MARIE orbital radiation data and MOLA laser altimetry. The lower the altitude, the lower the expected dose, because the atmosphere provides shielding. The global map of Mars on the left panel shows estimates for amounts of high-energy particle cosmic radiation reaching the surface of the planet. Colors in the map refer to the estimated average number of times per year each cell nucleus in a human there would be hit by a high-energy cosmic ray particle. The range is generally from two hits, a moderate risk level, to eight hits, a high risk level. The global map of Mars on the right panel shows the estimated radiation dose from GCRs reaching the surface. The colors in the map refer to the estimated annual dose equivalent in rems. The range varies from $10 \mathrm{rem} / \mathrm{yr}$ to $20 \mathrm{rem} / \mathrm{yr}$. This figure is courtesy of NASA/JPL/Johnson Space Center.

1997 to November 2006 [15]). The areas of Mars that are expected to have the lowest levels of cosmic radiation are at the lowest points of elevation, where there is more atmosphere above them, and at regions with a localized magnetic field.

All in all, using these measurements we estimate that the absorbed radiation dose during a round trip to Mars for a Hohmann transfer would be between $906 \mathrm{mSv}$ and 1,554 mSv, whereas the absorbed radiation dose during the stay on Mars would be between $97 \mathrm{mSv}$ and $115 \mathrm{mSv}$.

\section{Astronaut career radiation dose limits and prospects for space exploration}

Health risks from exposure to radiation on Earth in the form of X-ray or $\gamma$-ray radiation are well known. The primary concern is the development of cancer due to radiation induced mutations in DNA. DNA strand and tissue degradation, immunological changes, cataracts, and damage to the central nervous system are all serious risks that must also be mitigated in preparation for a manned mission to Mars. These health risks are likely amplified in space, where astronauts will face exposure to much higher energy radiation in the form of GCRs and SEPs.

To protect astronauts from the dangerous effects of high-energy radiation, the NASA spaceprogram has determined a set of career radiation exposure limits for astronauts. Career radiation exposure limits vary by age and are lower for younger astronauts. The logic behind this is that younger astronauts have a longer life to live and therefore a greater chance of developing subsequent health problems in their lifetime. Career radiation exposure limits also vary by sex and are lower for female astronauts. This is due to a number of factors, including a generally higher risk of radiation induced cancer in females.

The NCRP Report No. 98 (NCRP 1989) made recommendations pertaining to astronauts involved in space shuttle missions and the orbiting space station. The longer follow-up of the 
atomic bomb survivors and the evaluation of survivor organ doses by the DS86 dosimetry system, used to recommend dose to risk conversion factors, led to substantial increases in risk estimates [16]. The NCRP Report No. 132 (NCRP 2000) made new recommendations for radiation protection of astronauts to accommodate the results of [16]. The predictions of increased risk factors in the NCRP 2000 report are also supported by Mir space station microdosimetry measurements [17]. However, a few years after the publication of the NCRP Report No. 132, a reevaluation of atomic bomb survivor organ doses with longer follow-up of the cohort led to further increases in risk estimates [18]. In the last decade, NASA has deviated from the ground-based approach in which radiation weighting factors are adopted to assign equal risk quality factors for all types of cancer. In addition, the NCRP recommendation for use of sex and age at exposure-dependent limits was extended to consider a small specialized group of the population: a never-smoker model, which lowers radiation risk estimates by about $20 \%$ compared to estimates for a U.S. average population. The NSCR-2012 model yields another increment in risk estimates, with substantially weaker dependence on the age factor [19]. In Table 1 we show a comparison between the effective doses in the recent NSCR-2012 model and the values from NCRP Reports No. 98 and No. 132 [20]. (GCR solar modulation gives $\mathrm{a} \sim 5 \%$ effect [20].)

Setting side by side the most recent risk factors given in Table 1 and the absorbed dose estimates of Sec. 2 it is straightforward to see that (with current shielding technology) the Hohmann transfer is not suitable for a round trip to Mars. However, a long stay mission of about 550 days, with 900-day mission duration [4] would be feasible. Indeed, this is also consistent with the recent NASA twins multidimensional analysis, which allowed comparison of the impact of the spaceflight environment on one twin's human health onboard the International Space Station for 340 days (his twin served as a genetically matched ground control) to the simultaneous impact of the Earth environment [21]. The data suggest that human health can be sustained over a year-long spaceflight.

Table 1: Comparison of effective dose limits over $10 \mathrm{yr}$ period. The last row corresponds to never-smokers.

\begin{tabular}{c|cccc|cccc}
\hline \hline Sex & \multicolumn{4}{|c|}{ Female } & \multicolumn{4}{c}{ Male } \\
\hline Age (yr) & 25 & 35 & 45 & 55 & 25 & 35 & 45 & 55 \\
\hline NCRP 1989 & $1.00 \mathrm{~Sv}$ & $1.75 \mathrm{~Sv}$ & $2.50 \mathrm{~Sv}$ & $3.00 \mathrm{~Sv}$ & $1.50 \mathrm{~Sv}$ & $2.50 \mathrm{~Sv}$ & $3.25 \mathrm{~Sv}$ & $4.00 \mathrm{~Sv}$ \\
NCRP 2000 & $0.40 \mathrm{~Sv}$ & $0.52 \mathrm{~Sv}$ & $0.75 \mathrm{~Sv}$ & $1.35 \mathrm{~Sv}$ & $0.75 \mathrm{~Sv}$ & $1.00 \mathrm{~Sv}$ & $1.48 \mathrm{~Sv}$ & $2.98 \mathrm{~Sv}$ \\
NSCR 2012 & - & $0.48 \mathrm{~Sv}$ & $0.51 \mathrm{~Sv}$ & $0.59 \mathrm{~Sv}$ & - & $0.70 \mathrm{~Sv}$ & $0.75 \mathrm{~Sv}$ & $0.81 \mathrm{~Sv}$ \\
NSCR 2012 & - & $0.70 \mathrm{~Sv}$ & $0.75 \mathrm{~Sv}$ & $0.85 \mathrm{~Sv}$ & - & $0.79 \mathrm{~Sv}$ & $0.85 \mathrm{~Sv}$ & $1.15 \mathrm{~Sv}$ \\
\hline \hline
\end{tabular}

Given the perceived future of human spaceflight, there is a pressing need to build upon the understanding of protecting astronauts from HZE radiation. Heavier elements used in spacecraft shields actually produce more secondaries than lighter elements, such as carbon and hydrogen. This strongly supports the idea of looking into materials made out of lightweight elements. One attractive material is the lightweight polyethylene plastic called RFX1, which is $50 \%$ more effective at shielding solar flares and $15 \%$ more effective at shielding GCR radiation than aluminum is. However, while polyethylene plastic could improve the shielding, it is not strong enough for load bearing aerospace structural applications, which is why aluminum is primarily used. A new promising material under research at NASA is made out of boron, nitrogen, and hydrogen, and is called Boron Nitride Nanotubes (BNNT) [22]. Hydrogenated BNNT takes the form of microtubules - an 
excellent design for increased stability, mechanical strength, and resistance to extreme temperatures - and can also hold a large quantity of hydrogen atoms for even greater shielding. Hydrogenated BNNT microtubules can be woven into composites, fabric, yarn, and film forms that could be integrated into the spacecraft structure as well as the astronauts' spacesuits. While water is another molecule with a high hydrogen content and potential to absorb radiation, it is significantly heavier than polyethylene plastic and BNNT, and does not possess the necessary strength for structural applications. Utilizing water would require additional energy due to its mass and hence would be more cost intensive. Creating an electrostatic radiation shield around the spacecraft could also potentially deflect some cosmic radiation, but would still leave astronauts exposed to dangerous levels of radiation.

In future missions to Mars, longer/permanent stays on the planet would become a reality. Protective measures on the red planet could include constructing an underground shelter to reduce the amount of GCR radiation that penetrates into human tissues. Such an underground shelter would have to be built at $\sim 2,000 \mathrm{~g} / \mathrm{cm}^{2}$ depth for effective shielding of secondaries produced by the interaction of HZE particles with the atmosphere of Mars. There are several prospective lava tubes on Mars that are located in low lying regions of the planet and may be viable options for an underground shelter. As previously mentioned, lower elevations are locations with the least amount of cosmic radiation and the hollow lava tubes may extend well below the surface of Mars. Radiation experiments at analog lava tubes on Earth showed that, on average, the amount of radiation in the interior of the tubes is $82 \%$ lower than on the surface [23]. Given that Mars is a differentiated terrestrial planet that formed from analogous chondritic materials and that many of the same magmatic processes that took place on Earth happened on Mars as well, it is possible that the interior of lava tubes on Mars could also provide similar shielding from radiation. For example, the radiation level at the Hellas Planitia ( $342 \mu \mathrm{Sv} /$ day $)$ is considerably less than in other regions on the surface of Mars ( $\sim 547 \mu \mathrm{Sv} /$ day $)$. A radiation exposure of $342 \mu \mathrm{Sv} /$ day is, however, significantly higher than what humans are annually exposed to on Earth (levels typically range from about 1.5 to $3.5 \mathrm{mSv} / \mathrm{yr}$, but can be up to about $50 \mathrm{mSv} / \mathrm{yr}$ in some regions of Europe). Three candidate lava tubes identified in the vicinity of Hadriacus Mons (an ancient low-relief volcanic mountain along the northeastern edge of Hellas Planitia) could provide protection from excessive radiation exposure. The absorbed dose inside these natural caverns would be $\sim 61.64 \mu \mathrm{Sv} /$ day.

\section{Conclusions}

We have shown that with current technology sending a long stay time manned mission to Mars, with 180-day transfer to Mars and 180-day inbound transfer, could be feasible. Incorporating BNNT shielding into spacecrafts in conjunction with building underground shelter structures in lava tubes on Mars might be the key for colonization of the red planet. Further considerations should include investments in space weather architecture to detect the onset of SEP events and provide a sufficient warning for astronauts to seek additional protection/shelter.

\section{Acknowledgments}

This work has been supported by the U.S. National Science Foundation Grant PHY-2112257. 


\section{References}

[1] O. Jäkel, Z. Med. Phys. 14, 267 (2004).

[2] J. Barthel and N. Sarigul-Klijn, Acta Astronautica 144, 254 (2018).

[3] P. A. Zyla et al. [Particle Data Group], PTEP 2020, 083 C01 (2020).

[4] D. F. Landau and J. M. Longuski, J. Spacecraft and Rockets 43, 1036 (2006).

[5] L. A. Anchordoqui, Phys. Rept. 801, 1 (2019).

[6] H. Schoorlemmer, J. Hinton and R. López-Coto, Eur. Phys. J. C 79, 427 (2019).

[7] V. Florinski, G. P. Zank, and N. V. Pogorelov, J. Geophys. Res. 108, 1228 (2003).

[8] E. N. Parker, Planet. Space Sci. 13, 9 (1965).

[9] L. J. Gleeson and W. I. Axford, Astrophys. J. 154, 1011 (1968).

[10] V. Formisano, S. Atreya, T. Encrenaz, N. Ignatiev, and M. Giuranna, Science 306, 1758 (2004).

[11] C. Zeitlin et al., Science 340, 1080 (2013).

[12] J. Guo et al., Astron. Astrophys. 577, A58 (2015).

[13] D. M. Hassler et al., Science 343, 1244797 (2014).

[14] C. Zeitlin et al., Adv. Space Res. 33, 2204 (2004).

[15] D. E. Smith et al., Science 284, 1495 (1999).

[16] D. L. Preston and D. L. Pierce, Hiroshima, Japan: Radiation Effects Research Foundation; Report 9-87 (1987).

[17] F. A. Cucinotta, J. W. Wilson, J. R. Williams, and J. F. Dicello, Radiat. Meas. 132, 181 (2000).

[18] D. L. Preston, D. A. Pierce, Y. Shimizu, H. M. Cullings, S. Fujita, S. Funamoto, and K. Kodama, Radiat. Res. 162, 377 (2004).

[19] F. A. Cucinotta, M. Y. Kim, and L. Chappell, Washington, DC: National Aeronautics and Space Administration; NASA TP 2013-217375 (2013).

[20] F. A. Cucinotta, Health Phys. 108, 131 (2015).

[21] F. E. Garrett-Bakelman, Science 364, eaau8650 (2019).

[22] S. A. Thibeault, C. C. Fay, G. Sauti, J. H. Kang, and C. Park, Patent Application Publication US 2015/0248941 A1, (2015).

[23] A. J. Paris, E. T. Davis, L. Tognetti, and C. Zahniser, J. Wash. Acad. Sci. (in press) [arXiv:2004.13156]. 